This item was submitted to Loughborough's Research Repository by the author.

Items in Figshare are protected by copyright, with all rights reserved, unless otherwise indicated.

\title{
Foams built up by non-Newtonian polymeric solutions: Free drainage
}

PLEASE CITE THE PUBLISHED VERSION

http://dx.doi.org/10.1016/j.colsurfa.2016.07.097

PUBLISHER

(c) Elsevier

VERSION

AM (Accepted Manuscript)

PUBLISHER STATEMENT

This work is made available according to the conditions of the Creative Commons Attribution-NonCommercialNoDerivatives 4.0 International (CC BY-NC-ND 4.0) licence. Full details of this licence are available at: https://creativecommons.org/licenses/by-nc-nd/4.0/

\section{LICENCE}

CC BY-NC-ND 4.0

\section{REPOSITORY RECORD}

Arjmandi-Tash, Omid, Anna Trybala, Faiz M. Mahdi, Nina Kovalchuk, and Victor Starov. 2016. "Foams Built up by Non-newtonian Polymeric Solutions: Free Drainage". Loughborough University. https://hdl.handle.net/2134/24209. 
Elsevier Editorial system(tm) for Colloids and Surfaces A: Physicochemical and Engineering Aspects

Manuscript Draft

Manuscript Number:

Title: Foams Built Up by Non-Newtonian Polymeric Solutions: Free Drainage

Article Type: SI: Victor_Starov

Keywords: Foam Drainage, Free Drainage, Non-Newtonian Liquids, Polymeric Solutions

Corresponding Author: Prof. Victor Starov, MSc, PhD, DSC

Corresponding Author's Institution: Loughborough University

First Author: Victor Starov, MSc, PhD, DSC

Order of Authors: Victor Starov, MSc, PhD, DSc; Omid Arjmandi Tash; Anna Trybala, Dr; Faiz Mahdi, Dr; Nina Kovalchuk, Dr

Abstract: A mathematical model of free drainage of foam built up by a power-law non-Newtonian liquid is developed. The theory predictions are compared with the experimental data on the drainage of foams formed using commercially available AculynTM22 and AculynTM33 polymeric solutions. The rheological parameters of the polymeric solutions were independently measured and used in the calculations. The deduced dimensionless equations were solved using finite element method with appropriate boundary conditions. The numerical simulations show that the decrease in the foam height and liquid content is very fast in the very beginning of the drainage; however, it reaches a steady state at longer time. The predicted values of the time evolution of the foam height and liquid content are in good agreement with the measured experimental data. 


\section{Graphical Abstract}
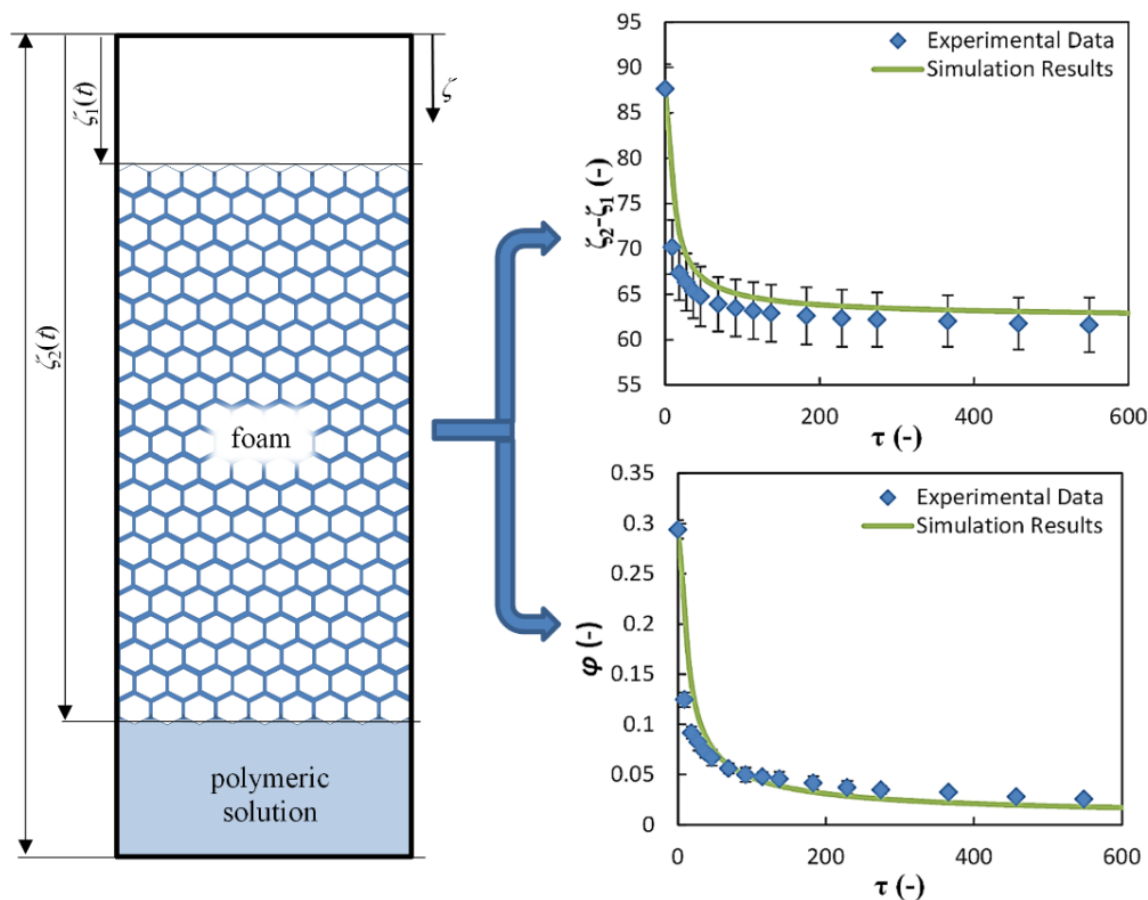
- A mathematical model of free drainage of foam is developed.

- Foam is built up by power-law non-Newtonian polymeric solutions.

- The rheological parameters of the polymeric solutions are independently measured.

- The predicted values are in good agreement with the measured experimental data. 


\title{
Foams Built Up by Non-Newtonian Polymeric Solutions: Free Drainage
}

\author{
O. Arjmandi-Tash ${ }^{1}$, A. Trybala ${ }^{1}$, F. M. Mahdi ${ }^{1}$, N. M. Kovalchuk ${ }^{1,2}$, V. $\operatorname{Starov}^{1}$ \\ ${ }^{1}$ Department of Chemical Engineering, Loughborough University, Loughborough, LE11 3TU, \\ UK \\ ${ }^{2}$ Institute of Biocolloid Chemistry, Kiev, Ukraine
}

\begin{abstract}
A mathematical model of free drainage of foam built up by a power-law non-Newtonian liquid is developed. The theory predictions are compared with the experimental data on the drainage of foams formed using commercially available Aculyn ${ }^{\mathrm{TM}} 22$ and Aculyn ${ }^{\mathrm{TM}_{33}}$ polymeric solutions. The rheological parameters of the polymeric solutions were independently measured and used in the calculations. The deduced dimensionless equations were solved using finite element method with appropriate boundary conditions. The numerical simulations show that the decrease in the foam height and liquid content is very fast in the very beginning of the drainage; however, it reaches a steady state at longer time. The predicted values of the time evolution of the foam height and liquid content are in good agreement with the measured experimental data.
\end{abstract}

Keywords: Foam Drainage, Free Drainage, Non-Newtonian Liquids, Polymeric Solutions 


\section{Introduction}

Foams are multiphase colloidal systems, which are formed by trapping a gas in a continuous phase (liquid or solid). Foams are widely used in food, pharmacy, personal care products, flotation and other separation processes, firefighting, petroleum and gas industries [1-3]. They pop up in lightweight mechanical materials and affect absorbing components in cars, heat exchangers and textured wallpapers.

A flow of liquid in between the gas bubbles through Plateau borders, nodes and films in foam driven by capillarity and/or gravity is referred to as drainage. In the case of foams built up by Newtonian liquids, equations of drainage were deduced using the combination of the liquid momentum and mass balance equations [4-8]. The drainage equations in the case of Newtonian liquids have been solved numerically and/or analytically in different prototype situations including free drainage [4-9], where liquid drains out of a foam due to the influence of gravity and capillarity; wetting of a dry foam $[10,11]$, where a dry foam is in contact with a liquid at its base; forced drainage [8, 9, 12-14], where liquid is added to the top of foam column producing a traveling wave; and pulsed drainage $[8,15-17]$, where a small volume of liquid is injected to the top of a foam and left to evolve. A new type of these situations is the case of foam drainage placed on a porous substrate $[18,19]$, where foam is deposited on a porous substrate and the presence of unsaturated pores inside the porous substrate results in an imbibition of liquid from the foam into the unsaturated pores.

Foams are conventionally stabilised by surfactants; however, polymers (polyelectrolytes) grow in popularity during the last decade as alternative stabilising additives to foaming solutions [20]. Polymers can adsorb at the interfaces and stabilize foam films like lowmolecular surfactants. The use of polymers in foaming solutions has found increasing benefits in different industrial applications. For example, polymer enhanced foams are 
increasingly being utilised for reservoir recovery in petroleum industry [21-23]. Polymers are also used in firefighting foams on polar fuels to prevent the foam from collapse [24]. In addition, polymer stabilized hair colouring foams were recently patented in Ref. [25] to provide improvements in colour delivery.

Understanding the rate of foam drainage/liquid release from foam is of great importance in various industries because it shows the rate of delivery of active components to the target places. Incorporating polymers into foaming solutions can affect the rate of drainage and thus, efficiency of the application. Addition of polymers often increases the viscosity of foaming solutions and therefore it affects the kinetics of foam drainage and the rate of inter-bubble gas diffusion [26-28]. For example, polymers used in firefighting foams can significantly lengthen the drainage time by viscosifying the aqueous phase. In general polymeric additives make the foaming solution shear thinning non-Newtonian fluids.

To date only a few foam drainage studies have been devoted to non-Newtonian fluids, such as polymer solutions [29, 30], while the majority of researches deal with Newtonian liquids, such as water, glycerin or oil. Despite intensive research in the field, to the best of our knowledge, only semi-empirical approaches have been developed so far and there is no comprehensive theory of foam drainage of non-Newtonian solutions. In our previous papers $[26,27,31]$ the influence of rheology of commercially available polymers $\operatorname{Aculyn}^{\mathrm{TM}} 22$ (A22) and Aculyn ${ }^{\mathrm{TM}} 33$ (A33) on the free foam drainage was investigated experimentally and the results of the properties modification (polymer type, concentration, mixtures, salt and isopropanol addition) of A22 and A33 polymeric solutions were presented. Below a theory of foam drainage is presented for the non-Newtonian polymeric solutions in the case of free drainage and its results are compared with experimental data. 


\section{Materials and methods}

A22 is a hydrophobically-modified alkali soluble emulsion (HASE) and A33 is an anionic alkali polymer emulsion, water soluble, lightly crosslinked. Polymer emulsions were supplied by Dow. Both polymers are soluble in water at high $\mathrm{pH}(\sim 12)$ and thus aqueous solutions of them were prepared by neutralising the stock polymer emulsions with a $2 \%$ ammonia solution in ultra-pure water produced by Millipore Q, with ascorbic and citric acids added ( 0.2 mass \% each). $\mathrm{NaCl}$ was added to solutions in the range of $0-1.3 \mathrm{M}$ concentrations. This composition represents the common system utilised in cosmetic products such as hair colourants where the ionic strength can be controlled by the added salt. The details about the preparation of polymeric solutions can be found in Refs. [26, 27].

The rheometer AR 1000-N, TA Instruments with the cone and plate geometry $(4 \mathrm{~cm}$ diameter, cone of $1^{\circ} 59^{\prime}$ and truncation of $56 \mu \mathrm{m}$ ) was used for the rheology measurements. Peltier plate was used to keep the temperature constant at $20^{\circ} \mathrm{C}$. In the oscillating mode the strain sweep measurements have been performed in the range of $0.1-10$ at frequency $1 \mathrm{~Hz}$ and in the flow mode shear rate measurements have been made at the values higher than $3 \mathrm{~s}^{-1}$ which corresponds to the local shear rates during the foam drainage [29]. The experimental error was lower than $5 \%$ at measuring probes taken from the same sample; however, the difference between individual samples of the same composition was higher, roughly $10 \%$.

Surface tension measurements have been performed by the Drop shape analyser DSA100, Kruss, Germany. Buoyant air bubble was formed at the tip of the hooked capillary immersed in the cuvette containing a polymer solution in these experiments.

Foaming experiments have been performed in a home-made glass column of $380 \mathrm{~mm}$ height and $43 \mathrm{~mm}$ inner diameter. The foaming head, fitted at the bottom of the column, consisted of 19 capillaries of $0.18 \mathrm{~mm}$ inner diameter for a gas supply. The foaming gas was 
air. To study the kinetics of foam drainage, a foaming liquid was poured into the column to the height of about $H_{0}=45 \mathrm{~mm}$ and bubbling was started at a constant flow rate. Bubbling was stopped after the foam height increased to about $150 \mathrm{~mm}$. Time evolution of the height of the foam, $\mathrm{H}_{2}-\mathrm{H}_{1}$ (Fig. 1) and the liquid under the foam, $\mathrm{H}-\mathrm{H}_{2}$, (Fig. 1) were measured during the experiments. The average liquid volume fraction inside the foam during the drainage was calculated as $\left(H_{0^{-}}\left(H-H_{2}\right)\right) /\left(H_{2}-H_{1}\right)$ and results of calculations are reported below. The produced foam was quite uniform (see Supplementary material Video 1) and the variation of bubble size due to coarsening was negligible during the drainage (bubble coarsening parameter $C$ was less than $10 \%$ for the solutions used in the experiments) [27]. Accordingly, an average bubble size was determined for each foam drainage experiment by analysing the pictures taken from the whole column during the drainage. Each experimental run was repeated at least twice and the average values are reported below. The details of foam drainage experiments can be found in Refs. [26, 27].

\section{Theory}

Let us consider foam composed of bubbles of uniform size and produced from polymeric solutions in a column of height $H$ (Fig. 1). Drainage occurs in the vertical direction along the co-ordinate axis $\mathrm{z}$ directed downward, with $\mathrm{z}=0$ at the top of the column. Time evolutions of the foam height and free liquid under the foam are $H_{2}(\mathrm{t})-H_{1}(\mathrm{t})$ and $H-H_{2}(\mathrm{t})$, respectively (Fig. 1). It is assumed below that the bubble size remains constant during the drainage, the dissipation occurs in the Plateau borders only and the contribution of the liquid flow in the films and nodes is neglected. 


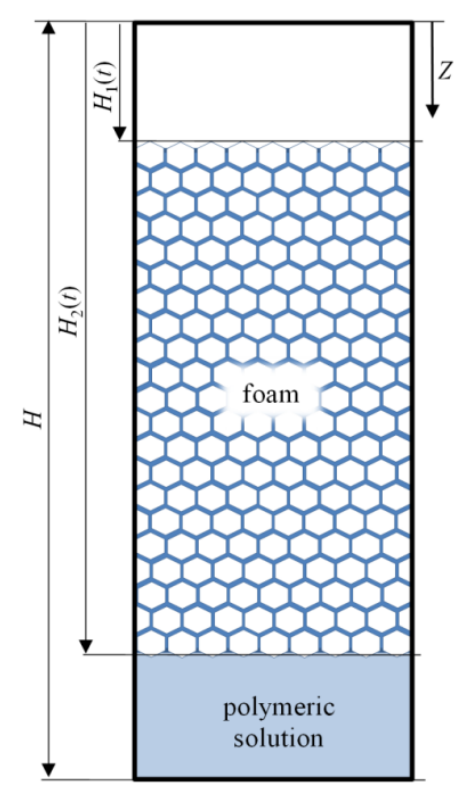

Fig. 1 Schematic of free foam drainage experiment

It was determined in Ref. [27] (and shown below) that the polymeric solutions of A22 and A33 are non-Newtonian power-law liquids, which show a shear-thinning behavior. For a power-law liquid dependence of the effective viscosity, $\mu_{\text {eff }}$, on applied shear rate is given by the well-known Ostwald-de Waele relation:

$$
\mu_{\text {eff }}=k \dot{\gamma}^{n-1}
$$

where $k$ is "flow consistency index", $\mathrm{n}$ is the "flow behavior index" and $\dot{\gamma}$ is the shear rate.

A velocity profile for flow of a power-law fluid in a pipe of radius $R$ with no-slip boundary condition is given by the following expression [32]:

$$
u(r)=\frac{n}{n+1}\left(\left|\frac{d P}{d z}\right| \frac{1}{2 k}\right)^{1 / n}\left(R^{1+1 / n}-r^{1+1 / n}\right)
$$

where $d P / d z$ is the pressure gradient and everywhere below we assume that the flow directed downward along z. According to Eq. 2, the average velocity, $V$, in a circular tube of crosssectional area of A, identical to that of actual Plateau border, is determined as follows: 


$$
V=\frac{n}{3 n+1} \frac{1}{\pi^{1 / 2+1 /(2 n)}}\left(\left|\frac{d P}{d z}\right| \frac{1}{2 k}\right)^{1 / n} A^{1 / 2+1 /(2 n)}
$$

According to Wang and Narsimhan, (2006) the average velocity in the actual Plateau border geometry, $v$, is related to that given by Eq. 3 as [33]:

$$
v=c V
$$

where the coefficient $c$ is [33]:

$$
c=a(n)
$$

In the above equation $a(n)$ are functions of flow behavior index, $n$, and their values are presented in Table 1. Here it is assumed that the interface is completely immobile (i.e. the Poiseuille flow).

Table. 1. The values of velocity coefficient in Eq. 5 for different values of flow behaviour index [33].

\begin{tabular}{|c|c|c|c|c|c|c|}
\hline$n$ & 1 & 0.8 & 0.6865 & 0.6 & 0.5 & 0.35 \\
\hline$a(n)$ & 0.5169 & 0.4851 & 0.459 & 0.434 & 0.3922 & 0.2942 \\
\hline
\end{tabular}

Substitution of Eq. 3 into Eq. 4 results in

$$
v=c \frac{n}{3 n+1} \frac{1}{\pi^{1 / 2+1 /(2 n)}}\left(\left|\frac{d P}{d z}\right| \frac{1}{2 k}\right)^{1 / n} A^{1 / 2+1 /(2 n)} .
$$

Hence, the flow rate, $q_{P b}$, in a Plateau border is as follows:

$$
q_{P b}=c \frac{n}{3 n+1} \frac{1}{\pi^{1 / 2+1 /(2 n)}}\left(\left|\frac{d P}{d z}\right| \frac{1}{2 k}\right)^{1 / n} A^{3 / 2+1 /(2 n)} .
$$


The quantity $P$ in Eqs. 2-7 is referred to modified pressure and it is an abbreviation for the sum of the capillary pressure and gravitational contributions. Accordingly, the driving force for Plateau border drainage $d P / d z$ is:

$$
\left|\frac{d P}{d z}\right|=\rho g+\frac{d}{d z}\left(\frac{\gamma}{R_{p b}}\right)
$$

where $\gamma$ is the liquid-air interfacial tension, $R_{p b}$ is the radius of curvature of Plateau border; $\rho$ and $g$ are the liquid density and the gravity acceleration, respectively. According to [34]:

$$
A=C^{2} R_{p b}^{2}=\frac{V_{b}}{n_{p} l} \frac{\varphi}{1-\varphi}=C_{1} R_{b}^{2} \frac{\varphi}{(1-\varphi)^{2 / 3}}
$$

where $\varphi$ is liquid volume fraction, $V_{b}$ is the volume of a bubble of radius $R_{b}, l$ is the length of the Plateau border, $n_{p}$ is the number of plateau borders per bubble; $\mathrm{C}^{2} \sim 0.161, C_{1}$ a geometrical coefficient, $C_{I}=4 \pi /\left(3 \mathrm{n}_{\mathrm{p}} \delta\right) \sim 0.378-0.972$ for a foam with structures between bcc (body-centred cubic) and fcc (face-centred cubic) and bubbles of the same size $\left(l=\delta R_{b}(1-\varphi)^{-}\right.$ ${ }^{1 / 3}, \delta=0.718-1.108, \mathrm{n}_{\mathrm{p}}=6-10$ ) [34]. Substituting Eqs. 9 and 8 into Eq. 7 results in the following equation for the flow rate in a Plateau border:

$$
q_{P b}=c \frac{n}{3 n+1} \frac{1}{\pi^{1 / 2+1 /(2 n)}} \frac{1}{2^{1 / n}}\left(\frac{\rho g}{k} A^{3 n / 2+1 / 2}-\frac{C \gamma}{2 k} A^{3 n / 2-1} \frac{\partial A}{\partial z}\right)^{1 / n} .
$$

If we define

$$
\frac{1}{f_{n}}=\left(c \frac{n}{3 n+1} \frac{1}{\pi^{1 / 2+1 /(2 n)}} \frac{1}{2^{1 / n}}\right)^{n}
$$

then Eq. 10 can be rewritten as

$$
q_{P b}=\left(\frac{\rho g}{k f_{n}} A^{3 n / 2+1 / 2}-\frac{C \gamma}{2 k f_{n}} A^{3 n / 2-1} \frac{\partial A}{\partial z}\right)^{1 / n} .
$$

According to Eq. 11 and Table $1, f_{n} \sim 49$ for a Newtonian liquid $(n=1)$ and immobile interface which is in complete agreement with the values reported earlier [8, 18, 35]. 
The total volumetric flux through the Plateau borders is therefore calculated as $[36,37]$ :

$$
Q=\frac{4}{15} N n_{p} R_{b}\left(\frac{\rho g}{k f_{n}} A^{3 n / 2+1 / 2}-\frac{C \gamma}{2 k f_{n}} A^{3 n / 2-1} \frac{\partial A}{\partial z}\right)^{1 / n}
$$

where $\mathrm{N}=(1-\varphi) / V_{b}$ is the number of bubbles per unit volume.

The mass conservation law for liquid inside Plateau border channels is [36]

$$
\frac{\partial \varphi}{\partial t}+\frac{\partial Q}{\partial z}=0
$$

Substituting Eq. 13 into Eq. 14 and replacing $A$ with expression 9 results in the following equation for the liquid volume fraction, $\varphi$ :

$$
\frac{\partial \varphi}{\partial t}+\frac{\partial}{\partial z}\left(\frac{C_{1}^{1 / 2} \rho g}{k \alpha_{n}} R_{b}^{n+1} \frac{\varphi^{3 n / 2+1 / 2}}{(1-\varphi)^{1 / 3}}-\frac{C \gamma}{2 k \alpha_{n}} R_{b}^{n} \frac{\varphi^{3 n / 2-1}(1-\varphi / 3)}{(1-\varphi)} \frac{\partial \varphi}{\partial z}\right)^{1 / n}=0
$$

where

$$
\frac{1}{\alpha_{n}}=\left(c \frac{n}{3 n+1} \frac{1}{2^{1 / n-3} 3^{3 / 2} 5} \frac{1}{n_{p}^{1 / 2} \delta^{3 / 2} \pi^{1 /(2 n)}}\right)^{n}
$$

Let us introduce the following dimensionless variable and co-ordinate:

$$
\zeta \rightarrow z / z_{0}, \quad \tau \rightarrow t / t_{0}
$$

where

$$
z_{0}=\sqrt{\frac{\gamma}{\rho g}}, t_{0}=\left(\frac{2 \alpha_{n} k}{C \gamma^{\frac{1-n}{2}} R_{b}^{n}(\rho g)^{\frac{n+1}{2}}}\right)^{1 / n} .
$$

Substitution of these variables into Eq. 15 results in

$$
\frac{\partial \varphi}{\partial \tau}+\frac{\partial}{\partial \zeta}\left(2 \sqrt{B o} \frac{\varphi^{3 n / 2+1 / 2}}{(1-\varphi)^{1 / 3}}-\frac{\varphi^{3 n / 2-1}(1-\varphi / 3)}{(1-\varphi)} \frac{\partial \varphi}{\partial \zeta}\right)^{1 / n}=0
$$

where 


$$
B o=\frac{\Delta p_{\text {grav }}}{\Delta p_{\text {cap }}}=\frac{\rho g \frac{C_{1}^{1 / 2}}{C} R_{b}}{\frac{\gamma}{C_{1}^{1 / 2}} R_{b}}=\frac{C_{1}}{C^{2}} \frac{\rho g R_{b}^{2}}{\gamma}
$$

is a corresponding Bond number.

For a fresh foam produced in the foam column, the liquid volume fraction profile is initially uniform, that is, $\varphi(\zeta, \tau=0)=\varphi_{i}[38,39]$.

\subsection{Boundary conditions}

During the drainage the liquid from the top of the foam drains to the lower parts. Therefore, the liquid volume fraction at the top decreases with time. There is a critical liquid volume fraction, $\varphi_{c r}$, below which the coalescence/bubble burst begins [40]. The value of this critical liquid volume fraction is a function of the nature of the surface active substances (polymer and/or surfactant) and their concentration. The critical liquid volume fraction is determined by a disjoining pressure action [36, 41, 42]. However, in Ref. [40] a mechanism based on a critical film dilatation is proposed for the onset of coalescence and critical liquid content in draining foams. Based on the theory of critical liquid volume fraction, there are two possible scenarios for the boundary condition at the top of the foam (i.e. $\mathrm{z}=H_{1}(\mathrm{t})$ or $\zeta_{=} \zeta_{1}(\mathrm{t})$ ): (i) If the liquid volume fraction at $\mathrm{z}=H_{1}$ is higher than $\varphi_{c r}$, then there is no bubble collapsing at the top of the foam (i.e. $d H_{1} / d t=0$ ) and the top boundary condition is zero liquid flux:

$$
Q\left(H_{1}, t\right)=0,
$$

or in dimensionless form:

$$
Q\left(\zeta_{1}, \tau\right)=0
$$


(ii) If the foam continues to dry and the liquid volume fraction at $\mathrm{z}=H_{1}$ drops to the value of $\varphi_{c r}$, then the bubbles at the top of the foam start to rupture and the height of the foam decreases from the top (i.e. $d H_{1} / d t>0$ ). In this case the boundary condition at the top of the foam is a constant liquid volume fraction:

$$
\varphi\left(H_{1}(t), t\right)=\varphi_{c r}
$$

or

$$
\varphi\left(\zeta_{1}(\tau), \tau\right)=\varphi_{c r}
$$

and the rate of foam collapse, $d H_{1} / d t$, can be expressed according to the following equation [36]:

$$
\frac{d H_{1}(t)}{d t}=\frac{1}{\varphi_{c r}} Q\left(H_{1}(t), t\right)
$$

or in dimensionless form:

$$
\frac{d \zeta_{1}(\tau)}{d \tau}=\frac{1}{\varphi_{c r}} Q\left(\zeta_{1}(\tau), \tau\right)
$$

After the onset of the drainage, the boundary condition at the bottom of the foam is a constant liquid volume fraction, $\varphi_{\max } \sim 0.36$, which corresponds to random packing limit for spherical particles in three dimensions [43]. However, as we consider a uniform initial liquid volume fraction along the foam height (i.e. $\varphi_{c r}<<\varphi(\zeta, \tau=0)=\varphi_{i}<\varphi_{\max }$ ), the boundary condition at the bottom of the foam should be zero liquid flux during a very early stage of the drainage until the liquid volume fraction at the bottom of the foam reaches $\varphi_{\max }$.

As the foam drains the polymeric solution accumulates under the foam and the interface between the foam and polymeric solution, $H_{2}$, moves up. The rate of this movement can be 
found using a mass conservation law of the polymeric solution within the whole column (see Supplementary material S1):

$$
\frac{d H_{2}(t)}{d t}=-\frac{1}{1-\varphi_{\max }} Q\left(H_{2}(t), t\right)
$$

or in dimensionless form:

$$
\frac{d \zeta_{2}(t)}{d \tau}=-\frac{1}{1-\varphi_{\max }} Q\left(\zeta_{2}(\tau), \tau\right)
$$

The above equations could be easily deduced from the fact that the product of the front velocity with the density difference of the two sides of the front is equal to the flux.

The equilibrium profile for liquid content inside the foam is reached when the gravity and capillary gradient forces equilibrate each other inside the foam. The equilibrium profile can be found by assuming zero liquid flux across the foam height. Integration of Eq. 15 in this case using the boundary condition at the bottom of the foam $\left(\varphi\left(H_{2}(t), t\right)=\varphi_{\max }\right)$ results in:

$$
\frac{2 C_{1}^{1 / 2} \rho g R_{b}}{C \gamma} \int_{z}^{H_{2 e}} \partial z=\int_{\varphi_{e q}}^{\varphi_{\max }} \varphi^{-3 / 2}(1-\varphi)^{-2 / 3}(1-\varphi / 3) \partial \varphi,
$$

and

$$
\left(1-\varphi_{e}(z)\right)^{1 / 3} \varphi_{e}^{-1 / 2}(z)=\left(1-\varphi_{\max }\right)^{1 / 3} \varphi_{\max }^{-1 / 2}+\frac{C_{1}^{1 / 2} \rho g R_{b}}{C \gamma}\left(H_{2 e}-z\right)
$$

Eq. 30 can be rewritten as the following dimensionless form:

$$
\left(1-\varphi_{e}(\zeta)\right)^{1 / 3} \varphi_{e}(\zeta)^{-1 / 2}=\left(1-\varphi_{\max }\right)^{1 / 3} \varphi_{\max }^{-1 / 2}+\sqrt{B o}\left(\zeta_{2 e}-\zeta\right)
$$

Eq. 31 shows that as expected, the equilibrium profile does not depend on flow behavior index, $n$, and it is identical to foams produced from Newtonian liquids. The calculated profile 
of the equilibrium liquid volume fraction is in good agreement with the results presented in Ref. [44] using the concept of an osmotic pressure (see Supplementary material Figure S1).

If $\zeta=\zeta_{1 e}$ is substituted in the above equation (we suppose that $\zeta_{1 e}$ is the dimensionless position of the top of the foam at equilibrium), then the equilibrium liquid volume fraction at the top of the foam can be deduced. The latter is a function of the foam height at equilibrium, $\zeta_{2 e}-\zeta_{1 e}$ and can be calculated as

$$
\left(1-\varphi_{e}\left(\zeta_{1 e}\right)\right)^{1 / 3} \varphi_{e}\left(\zeta_{1 e}\right)^{-1 / 2}=\left(1-\varphi_{\max }\right)^{1 / 3} \varphi_{\max }^{-1 / 2}+\sqrt{B o}\left(\zeta_{2 e}-\zeta_{1 e}\right)
$$

The dependency of the equilibrium liquid volume fraction at the top of the foam on the foam height (according to Eq. 32) is schematically illustrated in Fig. 2. As shown in Fig. 2, $\varphi_{e}\left(\zeta_{1 e}\right)$ increases to $\varphi_{\max }$ as the final foam height, $\zeta_{2 e}-\zeta_{1 e}$, decreases. Substituting $\varphi_{e}\left(\zeta_{1 e}\right)=\varphi_{c r}$ in Eq. 32 allows determining a critical foam height, $\lambda_{\mathrm{cr}}$. This critical foam height is shown in Fig. 2 where $\varphi_{e}\left(\zeta_{1 e}\right)=\varphi_{c r}$.

If the initial foam height, $\zeta_{2}(\tau=0)-\zeta_{1}(\tau=0)=\zeta_{2 i}-\zeta_{1 i}$ is less than $\lambda_{\mathrm{cr}}$ then over duration of the whole process the liquid volume fraction at the top of the foam remains above the $\varphi_{c r}$ and the top part of the foam does not move, that is $\zeta_{1}(\tau)=\zeta_{1 i}$. However, if $\zeta_{2 i}-\zeta_{1 i}$ is bigger than $\lambda_{\text {cr }}$ then two possibilities can be predicted: (1) over duration of the whole drainage process the liquid volume fraction at the top of the foam remains above the $\varphi_{c r}$, which is exactly the same as before, (2) at some moment in time, $\tau_{\mathrm{cr}}, \varphi\left(\zeta_{1}, \tau_{c r}\right)=\varphi_{c r}$. After that moment the apparent boundary of the foam at the top starts to decrease according to Eq. 26. 


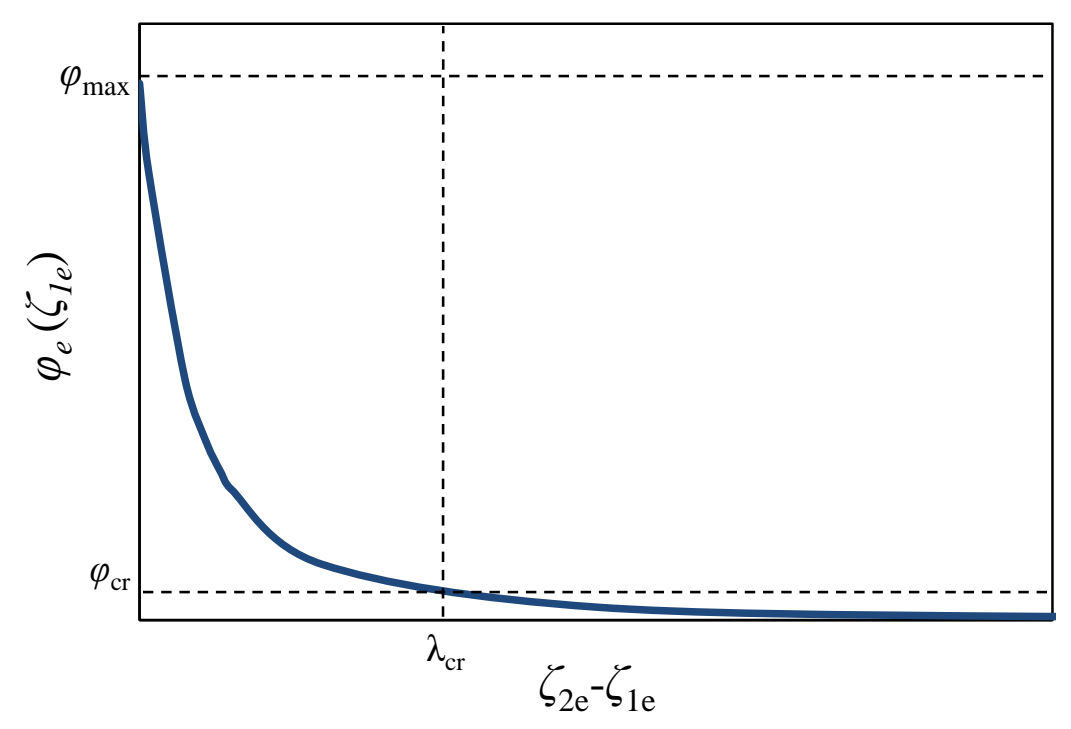

Fig. 2 The dependency of the equilibrium liquid volume fraction at the top of the foam on the foam height.

Therefore, the foam height will decrease from both top and bottom boundaries while $\varphi\left(\zeta_{1}, \tau\right)=\varphi_{c r}$. This reduction in foam height continues until the time when it reaches equilibrium. According to Eq. 32 and Fig. 2, the final height of the foam in this case is fixed and equal to the critical foam height, $\zeta_{2 e}-\zeta_{1 e}=\lambda_{c r}$. Considering a mass conservation of the polymeric solution within the whole column at initial and final state of the process, it is possible to predict which of the two above mentioned possibilities will occur:

$$
\varphi_{i}\left(H_{2 i}-H_{1 i}\right)+H-H_{2 i}=\int_{H_{1 e}}^{H_{2 e}} \varphi_{e}(H) d H+H-H_{2 e} .
$$

The left hand side of the equation shows the initial amount of the liquid presented within the whole column while the right hand side of the equation determines the final liquid content at equilibrium. Eq. 33 can be rewritten as the following dimensionless form: 


$$
\varphi_{i}\left(\zeta_{2 i}-\zeta_{1 i}\right)-\zeta_{2 i}=\int_{\zeta_{1 e}}^{\zeta_{2 e}} \varphi_{e}(\zeta) d \zeta-\zeta_{2 e}
$$

If we suppose that during the drainage the liquid volume fraction at the top of the foam drops to the value of $\varphi_{c r}$ (i.e. the second possibility occurs), then as mentioned above $\zeta_{2 e}=\zeta_{1 e}+\lambda_{c r}$. Substituting this expression into Eq. 34 results in

$$
\varphi_{i}\left(\zeta_{2 i}-\zeta_{1 i}\right)-\zeta_{2 i}=\int_{\zeta_{1 e}}^{\zeta_{1 e}+\lambda_{c r}} \varphi_{e}(\zeta) d \zeta-\left(\zeta_{1 e}+\lambda_{c r}\right)
$$

From the above equation

$$
\zeta_{1 e}-\zeta_{1 i}=\left(\zeta_{2 i}-\zeta_{1 i}\right)-\varphi_{i}\left(\zeta_{2 i}-\zeta_{1 i}\right)+\int_{\zeta_{1 e}}^{\zeta_{1 e}+\lambda_{c r}} \varphi_{e}(\zeta) d \zeta-\lambda_{c r}
$$

Since the foam height decreases from the top in this case, $\zeta_{1 e}-\zeta_{1 i}>0$ and the right hand side of Eq. 36 should be also a positive value:

$$
\left(\zeta_{2 i}-\zeta_{1 i}\right)-\varphi_{i}\left(\zeta_{2 i}-\zeta_{1 i}\right)+\int_{\zeta_{1 e}}^{\zeta_{1 e}+\lambda_{c r}} \varphi_{e}(\zeta) d \zeta-\lambda_{c r}>0
$$

According to the above equation the condition for the movement of the top of the foam during the drainage can be written as:

$$
\varphi_{i}<1-\frac{\left(\lambda_{c r}-\int_{\zeta_{1 e}}^{\zeta_{1 e}+\lambda_{c r}} \varphi_{e}(\zeta) d \zeta\right)}{\left(\zeta_{2 i}-\zeta_{1 i}\right)},
$$

or 


$$
\varphi_{i}<1-\frac{\left(\lambda_{c r}-\int_{0}^{\lambda_{c r}} \varphi_{e}\left(\zeta_{2 e}-\zeta\right) d \zeta\right)}{\left(\zeta_{2 i}-\zeta_{1 i}\right)}=\varphi_{t}
$$

A comparison between the values of $\varphi_{i}$ and $\varphi_{t}$ for each foaming solution can predict the state of the top of the foam during the drainage. The integral in Eq. 39 (to find the values of $\varphi_{t}$ ) can be calculated numerically using Eq. 31 for different values of Bo number and it is identical to the area under the equilibrium curve from $\zeta_{2 e}-\zeta_{1 e}=0$ to $\zeta_{2 e}-\zeta_{1 e}=\lambda_{c r}$ in Fig. 2. Therefore, in the case in which the initial foam height, $\zeta_{2 i}-\zeta_{1 i}$, is bigger than $\lambda_{\mathrm{cr}}$ the foam height decreases from the top boundary only if the condition specified in Eq. 39 is satisfied for the value of initial liquid volume fraction. Otherwise the liquid volume fraction at the top of the foam remains above the $\varphi_{c r}$ and the top boundary of the foam does not move.

\subsection{Model calculation}

The model of foam drainage described by dimensionless Eq. 19 has been solved using finite element method on one dimensional regular grid with 800-1200 elements corresponding to the foam height. A backward differentiation formula was used to solve time-dependent variables and time stepping was free taken by solver with initial step size of $10^{-20}$. Relative tolerance was set to $10^{-8}$, whereas absolute tolerance was set to $10^{-10}$. The boundary conditions at the top and bottom of the foam and their locations were imposed and updated as described in the previous section to obtain the evolution of liquid volume fraction inside the foam. The values of $t_{0}, z_{0}, B o, \lambda_{c r}$, flow behavior index, $n$, and flow consistency index, $k$, are obtained for different polymeric solutions based on the experimental data shown in the next section. 


\section{Results and discussion}

\subsection{Rheology and surface tension measurements of polymeric solutions}

The rheology of polymeric solution is an important parameter in the kinetic of the foam drainage. Fig. 3 shows the dependency of the measured effective viscosity of different polymeric solutions on shear rate. The dependencies are fitted according to Eq. 1. The obtained values of $k$ and $n$ for different polymeric solutions are listed in Table 2 and are used in calculations below. The shear thinning character of the polymeric solutions was found, as expected. Fig. 3(a) shows that the solutions viscosity decreases with increasing of the salt concentration. Fig. 3(b) also indicates that solution viscosity increases with the increase of the polymer concentration. The values of surface tension for different polymeric solutions are given in Table 2 and they are used in calculations.

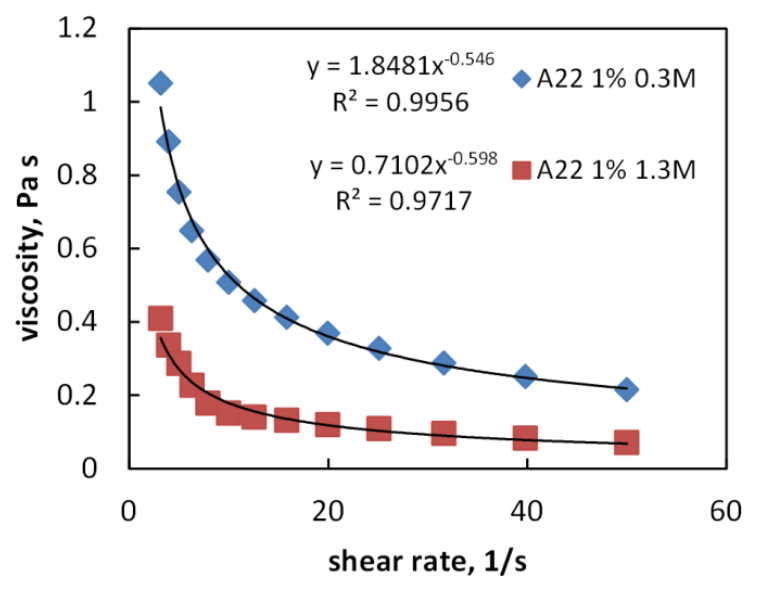

a)

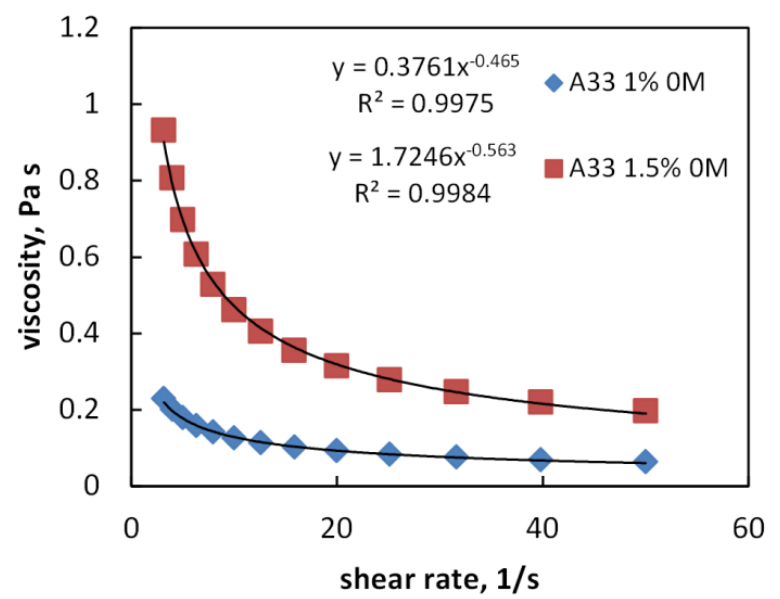

b)

Fig. 3 The dependency of the measured effective viscosity of a) A22 and b) A33 solutions on shear rate. The dependencies are fitted according to Eq. 1. Fitted values are given in Table 2. 


\subsection{Foam drainage of polymeric solutions}

Drainage of foams produced from power-law non Newtonian liquids of three compositions of A22 and A33 polymers have been chosen for investigation as shown in Table 2. The density of all solutions was around $1000 \mathrm{~kg} / \mathrm{m}^{3}$. Each foam drainage experiment was performed for 120 minutes. The average bubble size reported in Table 2 indicates that the foams formed from the more viscous solutions had larger bubbles. Based on the four obtained experimental data, $k, n, \gamma$ and $R_{b}$, we calculated $B o, c, \alpha_{n}, z_{0}, t_{0}$ and $\lambda_{c r}$ according to Eqs. 20, 5, 16, 18, 18 and 32 respectively, and their values for each foaming solutions are reported in Table 2. The values of velocity coefficient, $c$, were found according to Eq. 5 and Table 1. To find the critical foam height, $\lambda_{c r}$, (according to Eq. 32) the values of critical liquid volume fraction $\varphi_{c r}$, are required. In Ref. [40] a narrow field of liquid fraction ranging from 0.0005 to 0.0007 was found as the critical liquid volume fraction in foam stabilised by mixture of surfactants. As a rough estimate, this range was used below to calculate the critical foam height. As can be seen in Table 2, the initial foam height $(\sim 0.15 \mathrm{~m})$ is higher than the estimated critical values for different polymeric solutions. The values of $\varphi_{t}$ in Eq. 39 are also calculated for each foaming solution and their values are reported in Table 2. The initial liquid volume fraction, $\varphi_{i}(\sim 0.25-0.30)$ is lower than the calculated values of $\varphi_{t}$; thus, the condition specified in Eq. 39 is satisfied. Although this suggests that the liquid volume fraction at the top of the foam drops to the value of $\varphi_{c r}$ and the foam height decreases from the top boundary, the measured experimental data showed that the change of the location of the top of the foam, $H_{1}$, was negligible compared to the change of the foam height, $H_{2}-H_{1}$, for all polymeric solutions during the drainage (maximum $7.4 \%$ ). Foams produced from polymeric solutions are more stable than foams build up by surfactant solutions; thus, $\varphi_{c r}$ is expected to be lower and $\lambda_{\text {cr }}$ should be higher for foams stabilized by polymers than by 
surfactants only. It confirms that the liquid volume fraction at the top of the foam $\varphi\left(H_{1}, t\right)$ does not decrease to the value of $\varphi_{c r}$ during the draining and, hence, zero liquid flux boundary condition (Eqs. 21-22) was used in the simulations below. Accordingly, the value of $\varphi_{c r}$ is set so low that there is no collapse for the foams examined here. Our calculations according to Eq. 39 suggest a value of roughly 0.0001 for the critical liquid volume fraction $\varphi_{c r}$ within the foams produced from the polymeric solutions used in our experiments.

Table 2. Characteristic values of polymeric solutions and their foam drainage. The first four values, $k, n, \gamma$ and $R_{b}$ are extracted from the experimental data. The second seven values, $B o, c, \alpha_{n}, z_{0}, t_{0}, \lambda_{c r}$ and $\varphi_{t}$ are calculated according to Eqs. 20, 5, 16, 18, 18, 32 and 39 respectively.

\begin{tabular}{|c|c|c|c|c|c|c|c|c|c|c|c|}
\hline $\begin{array}{c}\text { Foaming } \\
\text { solutions }\end{array}$ & $\begin{array}{c}\mathrm{k} \\
\left(\mathrm{Pa} . \mathrm{s}^{\mathrm{n}}\right)\end{array}$ & $\begin{array}{c}\mathrm{n} \\
(-)\end{array}$ & $\begin{array}{c}\gamma \\
(\mathrm{N} / \mathrm{m})\end{array}$ & $\begin{array}{c}\mathrm{R}_{\mathrm{b}} \\
(\mathrm{m})\end{array}$ & $\begin{array}{c}\mathrm{Bo} \\
(-)\end{array}$ & $\begin{array}{c}\mathrm{c} \\
(-)\end{array}$ & $\begin{array}{c}\alpha_{\mathrm{n}} \\
(-)\end{array}$ & $\begin{array}{c}\mathrm{z}_{0} \\
(\mathrm{~m})\end{array}$ & $\begin{array}{c}\mathrm{t}_{0} \\
(\mathrm{~s})\end{array}$ & $\begin{array}{c}\lambda_{\text {cr }} \\
(-),(\mathrm{m})\end{array}$ & $\varphi_{t}$ \\
\hline A22_1.0\%1.3M & 0.7102 & 0.402 & 0.038 & 0.00139 & 3.003 & 0.334 & 20.575 & 0.00197 & 38.449 & $\begin{array}{c}20.976-24.973, \\
0.041-0.049\end{array}$ & $0.67-0.72$ \\
\hline A33_1.0\%0M & 0.3761 & 0.535 & 0.030 & 0.00131 & 3.358 & 0.408 & 31.033 & 0.00175 & 13.115 & $\begin{array}{c}19.837-23.617, \\
0.035-0.041\end{array}$ & $0.73-0.78$ \\
\hline A33_1.5\%0M & 1.7246 & 0.437 & 0.027 & 0.00158 & 5.435 & 0.358 & 22.916 & 0.00166 & 316.148 & $15.593-18.564$, & $0.026-0.031$ \\
\hline
\end{tabular}

Figure 4 shows calculated results on the time evolution of liquid volume fraction, $\varphi$, over the foam height, $\zeta$, for "A33_1.0\% 0M" solution. In this figure $\zeta=0$ is the top of the foam and $\varphi_{\mathrm{i}}=0.2939$, according to the experimental data. In the very beginning of the drainage the liquid volume fraction varies only at the top and bottom of the foam, whereas in the middle part of the foam the initial value is retained. The interface between the foam and polymeric solution at the bottom is moving up during the drainage. This decrease of the foam height is 
very fast in the early stage of the drainage; however, reduction of the foam height and the profile of the liquid volume fraction approach a steady state over time.

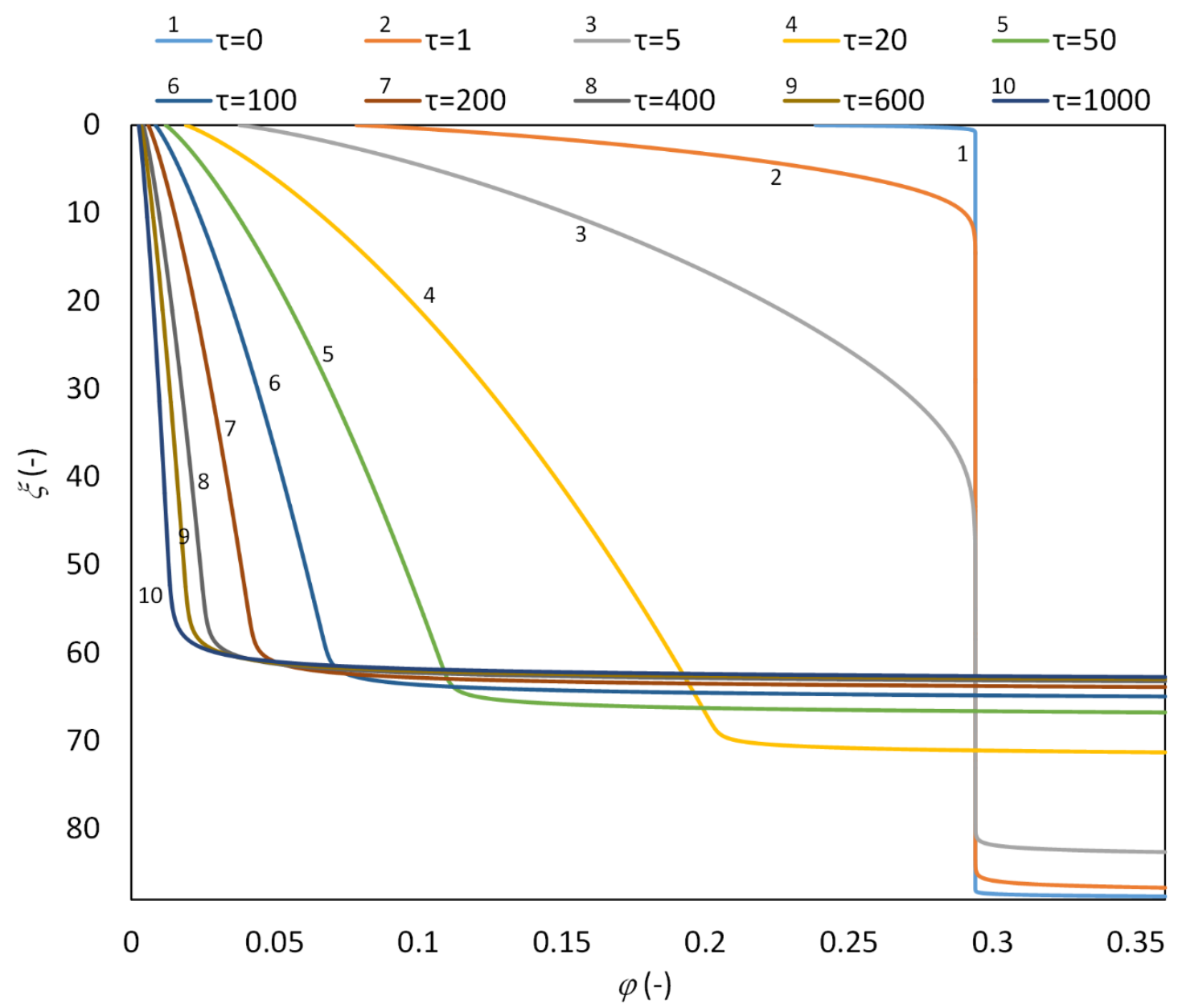

Fig. 4 Time evolution of the predicted results of liquid volume fraction, $\varphi$, over the foam height, $\zeta$, for "A33_1.0\% 0M" solution ( $\zeta=0$ corresponds to the top of the foam).

Comparison of the predicted and experimental time evolution of the height of the foam, $\zeta_{2}-\zeta_{1}$, and average liquid volume fraction, $\varphi$, is shown in Figs. 5 and 6, respectively for different polymeric solutions. As expected, for all polymeric solutions the foam height and the average liquid volume fraction decrease dramatically in the beginning of the drainage and after some time they reach a plateau. As can be seen in Figs. 5 and 6, the predicted values are in a quantitative agreement with the measured experimental data $\left(\mathrm{R}^{2}>0.85\right)$. According to Ref. [29] 
elongational properties of the polymeric solution can also affect the foam drainage. It was found that the rate of the drainage increases by increasing the elongational viscosity of the solutions. This influence can be important at higher contractions of the polymeric solutions and with larger polymer molecular weights [29, 30], this phenomenon could account for the small deviations between predicted results and experimental data presented below.
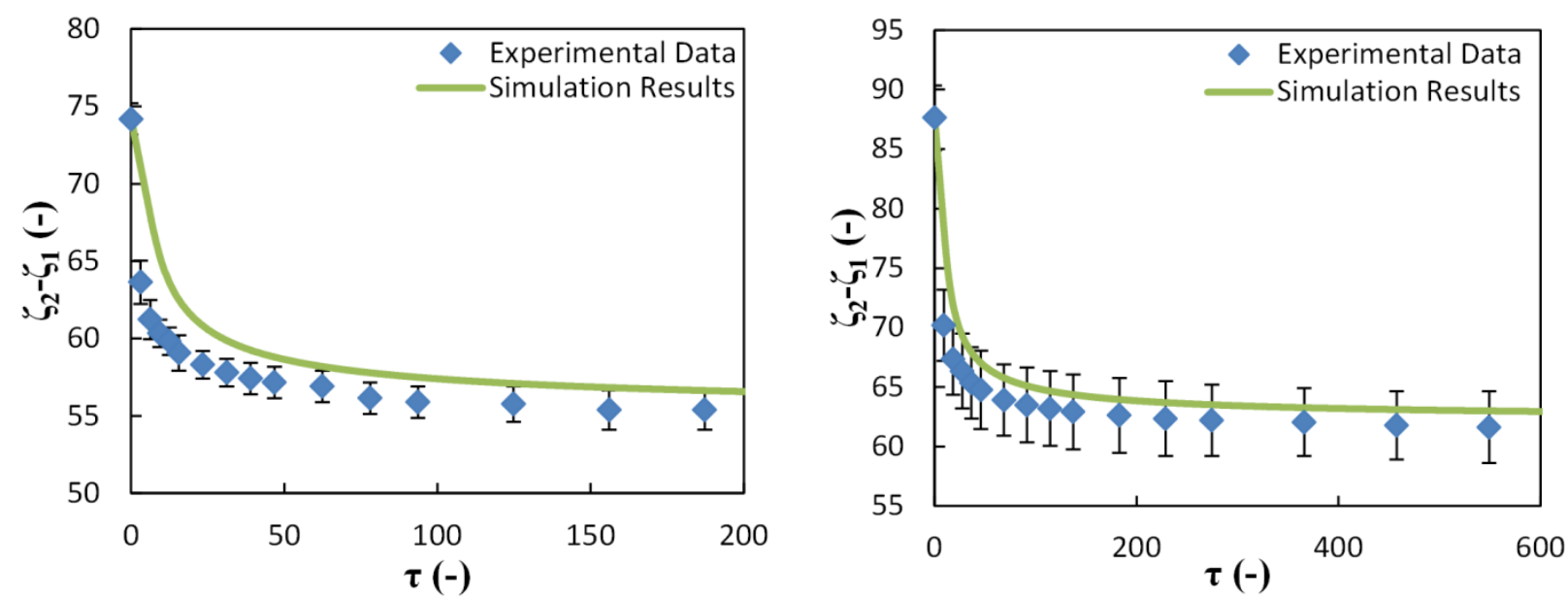

a)

b)

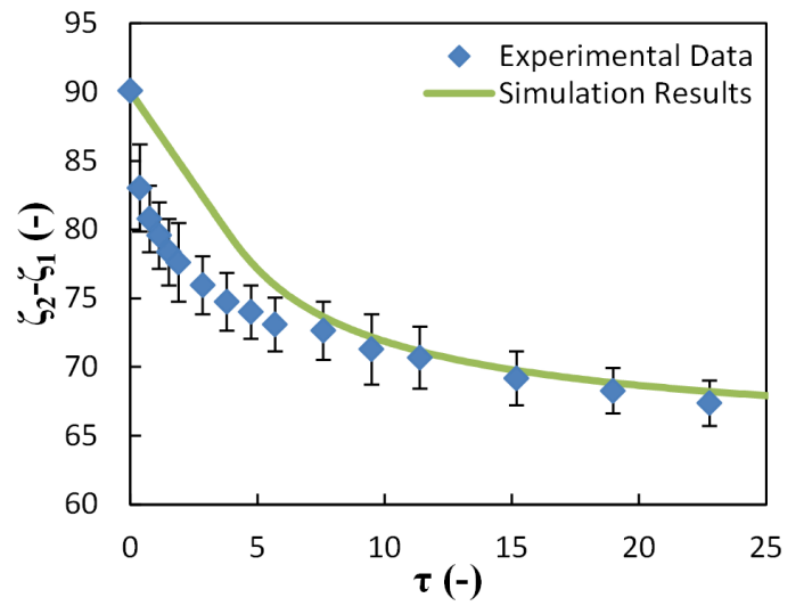

c)

Fig. 5 Comparison of the predicted time evolution of the height of the foam, $\zeta_{2}-\zeta_{1}$, with experimental data for a) A22_1.0\% 1.3M, b) A33_1.0\% 0M and c) A33_1.5\% 0M. 

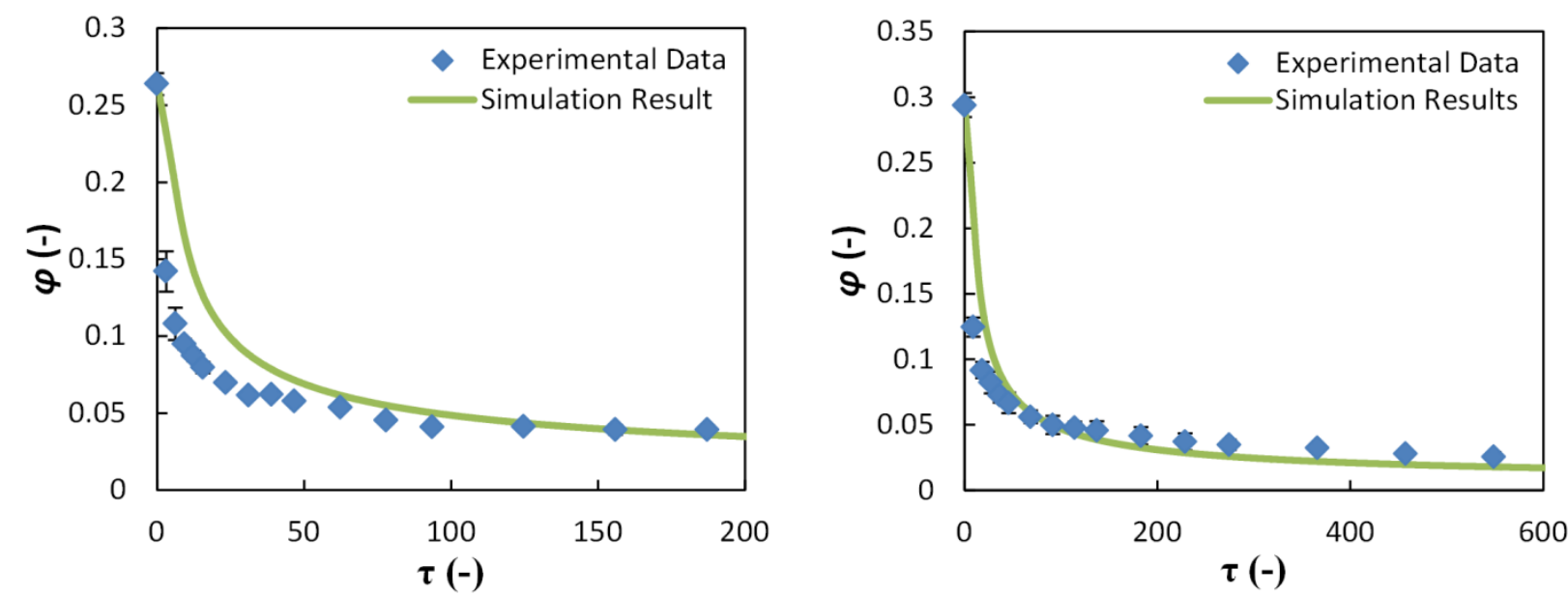

a)

b)

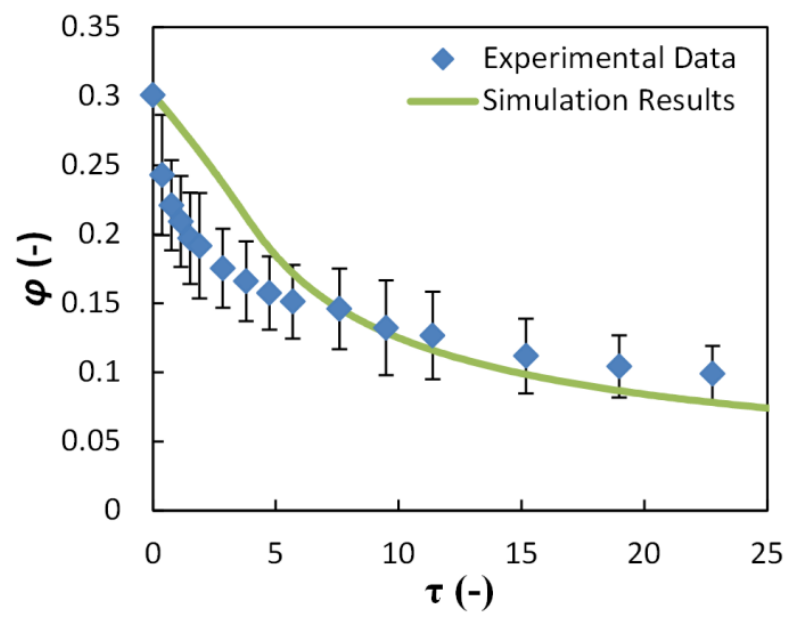

c)

Fig. 6 Comparison of the predicted time evolution of the average liquid volume fraction along the foam height, $\varphi$, with experimental data for a) A22_1.0\% 1.3M, b) A33_1.0\% 0M and c) A33_1.5\% 0M.

\section{Conclusions}

A model for free drainage of foams produced from power-law non-Newtonian shear thinning liquids is presented and compared with experimental data for the drainage of foams formed by A22 and A33 polymeric solutions. Equation was deduced, which describes the drainage of foam build up by a power-law non-Newtonian liquid. The boundary condition at foam/liquid interface is a constant liquid volume fraction corresponding to the maximum liquid volume 
fraction inside the foam. There are two possible boundary conditions at the top of the foam which depends on the initial foam height and initial liquid content within the foam: (i) If the liquid content at the top is higher than a critical liquid volume fraction, there is no collapsing of bubbles at the top of the foam and boundary condition is zero liquid flux; (ii) If the liquid volume fraction at the top of the foam drops to a critical value, the height of the foam decreases from the top by collapsing of the bubbles at the top of the foam. The predicted values showed that in the early stage of the drainage the liquid content varies only at the top and bottom of the foam, whereas in the middle section of the foam the initial value is retained. The interface between the foam and polymeric solution was moving up during the drainage and the kinetics of this motion is predicted. This decrease in the foam height was very fast in the very beginning of the drainage; however, it reached a steady state over time. The equilibrium profile of the liquid volume fraction is predicted and shown that it does not depend on power law index, $\mathrm{n}$, of non-Newtonian liquid. The predicted values of the time evolution of the foam height and liquid content were in good agreement with the experimental data.

\section{Acknowledgements}

This research was supported by EU CoWet project; Procter \& Gamble, USA; EPSRC, UK, grant EP/J010596/1; PASTA and MAP EVAPORATION projects, European Space Agency; and COST project MP1106.

\section{Nomenclature}




\begin{tabular}{|c|c|}
\hline$a$ & velocity coefficient \\
\hline$A$ & Plateau border cross-sectional area, $\mathrm{m}^{2}$ \\
\hline Bo & Bond number \\
\hline$c$ & velocity coefficient \\
\hline$C$ & geometrical coefficient \\
\hline$C_{1}$ & geometrical coefficient \\
\hline$f_{n}$ & drag coefficient \\
\hline g & gravity acceleration, $\mathrm{m} / \mathrm{s}^{2}$ \\
\hline$H$ & column height, $\mathrm{m}$ \\
\hline$H_{0}$ & initial height of the liquid before producing the foam, $\mathrm{m}$ \\
\hline$H_{1}$ & position of the top of the foam, $m$ \\
\hline$H_{1 e}$ & position of the top of the foam at equilibrium, $m$ \\
\hline$H_{1 i}$ & initial position of the top of the foam, $\mathrm{m}$ \\
\hline $\mathrm{H}_{2}$ & position of the foam/polymeric solution boundary, $\mathrm{m}$ \\
\hline$H_{2 e}$ & position of the foam/polymeric solution boundary at equilibrium, $\mathrm{m}$ \\
\hline$H_{2 i}$ & initial position of the foam/polymeric solution boundary, $\mathrm{m}$ \\
\hline$k$ & flow consistency index, $\mathrm{Pa} \mathrm{s}^{\mathrm{n}}$ \\
\hline$l$ & length of the Plateau border, $\mathrm{m}$ \\
\hline
\end{tabular}


$n_{p} \quad$ number of plateau borders per bubble

$N \quad$ number of bubbles per unit volume, $1 / \mathrm{m}^{3}$

$p \quad$ pressure, $\mathrm{Pa}$

$P \quad$ modified pressure, $\mathrm{Pa}$

$q_{P b} \quad$ flow rate in Plateau border, $\mathrm{m}^{3} / \mathrm{s}$

$Q \quad$ total volumetric flux through the Plateau borders, $\mathrm{m} / \mathrm{s}$

$r \quad$ radial co-ordinate, $\mathrm{m}$

$R \quad$ radius, $\mathrm{m}$

$R_{b} \quad$ radius of bubbles, $\mathrm{m}$

$R_{P b} \quad$ curvature radius of Plateau border, $\mathrm{m}$

$t \quad$ time, $\mathrm{s}$

$t_{0} \quad$ characteristic time scale, $\mathrm{s}$

$u \quad$ velocity, $\mathrm{m} / \mathrm{s}$

$V \quad$ average velocity in a circular tube, $\mathrm{m} / \mathrm{s}$

$V_{b} \quad$ bubble volume, $\mathrm{m}^{3}$

$z \quad$ co-ordinate axis, $\mathrm{m}$

$z_{0} \quad$ characteristic length scale, $\mathrm{m}$ 


\begin{tabular}{|c|c|}
\hline$\gamma$ & surface tension, $\mathrm{N} / \mathrm{m}$ \\
\hline$\dot{\gamma}$ & shear rate, $\mathrm{s}^{-1}$ \\
\hline$\delta$ & geometrical coefficient \\
\hline$\zeta$ & dimensionless vertical co-ordinate \\
\hline$\zeta_{1}$ & dimensionless position of the top of the foam \\
\hline$\zeta_{l e}$ & dimensionless position of the top of the foam at equilibrium \\
\hline$\zeta_{1 i}$ & dimensionless initial position of the top of the foam \\
\hline$\zeta_{2}$ & dimensionless position of the foam/polymeric solution boundary \\
\hline$\zeta_{2 e}$ & $\begin{array}{l}\text { dimensionless position of the foam/polymeric solution boundary at } \\
\text { equilibrium }\end{array}$ \\
\hline$\zeta_{2 i}$ & dimensionless initial position of the foam/polymeric solution boundary \\
\hline$\lambda_{c r}$ & dimensionless critical foam height \\
\hline$\mu_{\text {eff }}$ & effective viscosity, Pa s \\
\hline$v$ & average velocity in actual Plateau border geometry, $\mathrm{m} / \mathrm{s}$ \\
\hline$\rho$ & liquid density, $\mathrm{kg} / \mathrm{m}^{3}$ \\
\hline$\tau$ & dimensionless time \\
\hline$\varphi$ & olume \\
\hline
\end{tabular}



$\varphi_{1}$
initial liquid volume fraction
$\varphi_{\mathrm{cr}}$
critical liquid volume fraction
$\varphi_{e}$
equilibrium liquid volume fraction
$\varphi_{\max }$
maximum liquid volume fraction

\section{References}

[1] D. Exerowa and P. M. Kruglyakov, Foams and foam films: Theory, experiment, application, Elsevier, Amsterdam, 1998.

[2] S. Farrokhpay, Advances in Colloid and Interface Science, 2011, 166, 1-7.

[3] A. Arzhavitina and H. Steckel, International Journal of Pharmaceutics, 2010, 394, 1-17.

[4] J. B. M. Hudales and H. N. Stein, Journal of Colloid and Interface Science, 1990, 140, 307-313.

[5] M. V. Ramani, R. Kumar and K. S. Gandhi, Chemical Engineering Science, 1993, 48, 455-465.

[6] A. R. Bhakta and K. C. Khilar, Langmuir, 1991, 7, 1827-1832.

[7] G. Verbist and D. L. Weaire, EPL (Europhysics Letters), 1994, 26, 631.

[8] G. Verbist, D. L. Weaire and A. M. Kraynik, Journal of Physics: Condensed Matter, 1996, 8, 3715-3731.

[9] S. Jun, D. D. Pelot and A. L. Yarin, Langmuir, 2012, 28, 5323-5330. 
[10] S. A. Koehler, H. A. Stone, M. P. Brenner and J. Eggers, Physical Review E, 1998, 58, 2097.

[11] Q. Sun, L. Tan and G. Wang, International Journal of Modern Physics B, 2008, 22, 2333-2354.

[12] D. L. Weaire, N. Pittet, S. Hutzler and D. Pardal, Physical Review Letters, 1993, 71, 2670.

[13] S. Hutzler and D. L. Weaire, Philosophical Magazine Letters, 2000, 80, 419-425.

[14] P. Stevenson, Colloids and Surfaces A: Physicochemical and Engineering Aspects, 2007, $305,1-9$.

[15] S. A. Koehler, S. Hilgenfeldt and H. A. Stone, Langmuir, 2000, 16, 6327-6341.

[16] S. A. Koehler, S. Hilgenfeldt and H. A. Stone, EPL (Europhysics Letters), 2001, 54, 335.

[17] S. A. Koehler, S. Hilgenfeldt and H. A. Stone, American Physical Society, Division of Fluid Dynamics Meeting, New Orleans, USA, 1999.

[18] O. Arjmandi-Tash, N. Kovalchuk, A. Trybala and V. Starov, Soft matter, 2015, 11, 3643-3652.

[19] A. Bureiko, O. Arjmandi-Tash, N. Kovalchuk, A. Trybala and V. Starov, The European Physical Journal Special Topics, 2015, 224, 459-471.

[20] N. Kristen and R. von Klitzing, Soft Matter, 2010, 6, 849-861.

[21] D. S. R. Sarma, J. Pandit and K. C. Khilar, Journal of Colloid and Interface Science, 1988, 124, 339-348. 
[22] L. L Schramm, Surfactants: fundamentals and applications in the petroleum industry; Cambridge University Press, 2000.

[23] S. E. Friberg and J. Fang, Journal of Colloid and Interface Science, 1987, 118, 543-552.

[24] P. Stevenson, Foam engineering: fundamentals and applications, John Wiley \& Sons, 2012.

[25] B. S. Lane, F. Vohra, S. K. Galazka, K. M. Nally, E. H. Agostino, G. S. Kerr, R. D. Lewis, M. T. Lund, S. D. McConaughy and E. D. Smith III, 2012.

[26] A. Bureiko, A. Trybala, J. Huang, N. Kovalchuk and V. Starov, Colloids and Surfaces A: Physicochemical and Engineering Aspects, 2013, 434, 268-275.

[27] A. Bureiko, A. Trybala, J. Huang, N. Kovalchuk and V. Starov, Colloids and Surfaces A: Physicochemical and Engineering Aspects, 2014, 460, 265-271.

[28] R. V. Klitzing and H. Müller, Current Opinion in Colloid \& Interface Science, 2002, 7, $42-49$.

[29] M. Safouane, A. Saint-Jalmes, V. Bergeron and D. Langevin, The European Physical Journal E, 2006, 19, 195-202.

[30] M. Safouane, M. Durand, A. Saint Jalmes, D. Langevin and V. Bergeron, Le Journal de Physique IV, 2001, 11, Pr6-275-Pr6-280.

[31] A. Bureiko, A. Trybala, N. Kovalchuk and V. Starov, Advances in Colloid and Interface Science, 2015, 222, 670-677.

[32] R. B. Bird, W. E. Stewart and E. N. Lightfoot, Transport phenomena, John Wiley \& Sons, 2007. 
[33] Z. Wang and G. Narsimhan, Journal of Colloid and Interface Science, 2006, 300, 327337.

[34] D. L. Weaire and S. Hutzler, The physics of foams; Oxford University Press, 1999.

[35] P. M. Kruglyakov, S. I. Karakashev, A. V. Nguyen and N. G. Vilkova, Current Opinion in Colloid \& Interface Science, 2008, 13, 163-170.

[36] A. Bhakta and E. Ruckenstein, Advances in Colloid and Interface Science, 1997, 70, 1124.

[37] G. Narsimhan, Journal of Food Engineering, 1991, 14, 139-165.

[38] S. Magrabi, B. Dlugogorski and G. Jameson, AIChE Journal, 2001, 47, 314-327.

[39] S. Magrabi, B. Dlugogorski and G. Jameson, Chemical Engineering Science,1999, 54, 4007-4022.

[40] V. Carrier and A. Colin, Langmuir, 2003, 19, 4535-4538.

[41] J. Bibette, D. Morse, T. Witten and D. Weitz, Physical Review Letter, 1992, 69, 2439.

[42] K. Khristov, D. Exerowa and G. Minkov, Colloids and Surfaces A: Physicochemical and Engineering Aspects, 2002, 210, 159-166.

[43] M. Van Hecke, Journal of Physics: Condensed Matter, 2010, 22, 033101.

[44] A. Maestro, W. Drenckhan, E. Rio and R. Höhler, Soft Matter, 2013, 9, 2531-2540. 
Supplementary Material
Click here to download Supplementary Material: Supplementary material.docx

Supplementary Material
Click here to download Supplementary Material: Supplementary material.docx

$x$

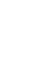

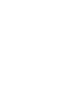

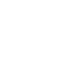

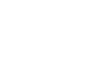

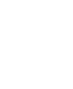

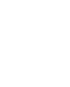

.

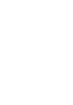

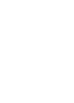

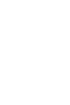

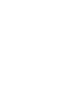

.

.

.

. 更

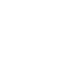

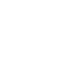

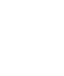
更

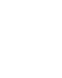

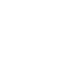

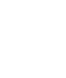

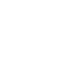

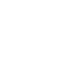

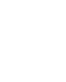
更

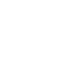

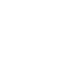

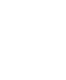

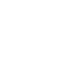


Supplementary Material
Click here to download Supplementary Material: Video 1.avi

+ 
A mathematical model of free drainage of foam built up by a power-law non-Newtonian liquid is developed. The theory predictions are compared with the experimental data on the drainage of foams formed using commercially available Aculyn ${ }^{\mathrm{TM}} 22$ and Aculyn ${ }^{\mathrm{TM}} 33$ polymeric solutions. The rheological parameters of the polymeric solutions were independently measured and used in the calculations. The deduced dimensionless equations were solved using finite element method with appropriate boundary conditions. The numerical simulations show that the decrease in the foam height and liquid content is very fast in the very beginning of the drainage; however, it reaches a steady state at longer time. The predicted values of the time evolution of the foam height and liquid content are in good agreement with the measured experimental data. 